\title{
New Inequality and Functional for Contact with Friction: The Implicit Standard Material Approach
}

\section{G. De Saxce}

Department of Structural Mechanics

Polytechnic Faculty of Mons

Mons, Belgium

\section{Z. Q. Feng}

Department of Mechanical Engineering UNIVERSITY OF TECHNOLOGY OF COMPIEGNE Compiegne, France

\section{ABSTRACT}

This paper is devoted to the analysis of the two- or three-dimensional elastic contact problem with Coulomb friction, quasi-static equilibrium, and small displacements. The classical approach is based on two minimum principles, or variational inequalities: the first for unilateral contact and the second for friction. In practical applications, this leads to an algorithm of alternately solving the two problems until convergence is achieved. A coupled approach using one principle or one inequality only is presented. This new approach, based on a model of material called implicit standard, allows for extension of the notion of a normality law to dissipative be- 
havior with a nonassociated flow rule, such as surface friction. For numerical time integration of the laws, Moreau's implicit method is considered. Nondifferentiable potentials are regularized by means of the augmented Lagrangian technique. A discretized formulation using the finite element method and numerical applications are reported in a separate paper.

\section{INTRODUCTION}

During the two last decades, many papers dealing with contact problems using the finite element method have been written. In this work, attention is focused on modeling unilateral contact with Coulomb friction and small displacements in order to obtain variational formulations, minimization or inequality problems. Before proceeding, three relevant features of contact problems must be stated.

First, as the sliding law involves time rates, an integration method must be chosen to determine the solution by numerical analysis. An explicit scheme is not satisfactory because the friction criterion is not fulfilled at the end of the step. As it is not possible to control the distance of a given traction state to Coulomb's cone, errors accumulate at the end of the loading steps. In the present approach, an implicit integration method is used. Although it is more complicated, it always gives better results concerning convergence and numerical stability [1-4]. For the elastoplastic law, the implicit method has been discussed by Moreau [3] and is known as the catching-up algorithm.

Second, the contact law is written in a local frame that depends on the unit vector normal to the contact surface in the configuration at the end of the step. A fully implicit method that takes into account this fact was proposed by Wriggers, Simo, and Taylor $[5,6]$ and Curnier and Alart $[7,8]$. Using small displacement hypotheses, such a degree of accuracy of the solution seems unnecessary. Hence, in the present approach, the normal unit vector is a priori determined by an assumption concerning possible contact. In this sense, the integration method can be characterized as semi-implicit [9].

Third, dry friction behavior is a dissipative process that is similar to a rigid-perfectly plastic law. Coulomb's criterion is analogous to the plasticity criterion and the sliding rule, to the yielding law. A large range of plastic behaviors, or more generally of dissipative processes, may be presented by a normality law [10-18]. This class of material is called generalized standard materials (GSM) [16]. This kind of material exhibits good properties with respect to the existence of solutions for the boundary-value problem. Because the dry friction sliding rule is a normality law where the derivative with respect to the normal traction is missing, the contact behavior may be 
considered nonstandard. Other plasticity laws in metals [19] or soils $[1,20$, 21] also can be called nonstandard material laws. In this paper, a new class of materials, called implicit standard materials, is presented, which can be used to generalize in a simple, neat way the standard materials and to define several interesting properties for the boundary-value problem.

A good generalization should preserve the notion of energetic law defined by the Legendre transform. The classical standard materials are determined by the knowledge of two dual potentials and an explicit dependence of the generalized stresses or forces with respect to the generalized strains or displacements.

The implicit standard materials are determined by a unique potential called a bipotential, and the dependence between generalized stresses and strains is implicit (in the sense of the implicit function theorem). By introduction of the so-called left and right dual potentials, it is shown that, with convexity assumptions, the implicit dependence can become explicit, but with the drawback that the standard form of the material law is lost. Some properties of existence of solutions are proved for boundary-value problems with implicit standard materials by introduction of variational principles based on the so-called "bifunctional." This approach leads to an iterative algorithm to reach the solution.

For unilateral contact with friction between elastic bodies, the new material law model leads to a single displacement variational principle for which the unilateral contact and the fricton law are coupled. This variational approach is simpler than the classical one, which involves two variational principles, one for unilateral contact and the other for friction [7, 8, 12, 22]:

When displacements are expressed with respect to traction, as in global GSM theory [18], the problem may be presented in inequality form. For the classical approach, two inequalities are obtained, which must be alternately solved until convergence $[9,12,23-25]$. For the implicit standard material approach, a unique inequality is obtained because unilateral contact and friction are coupled.

For the numerical solution process, the augmented Lagrangian method [26] is used in order to avoid occurrence of nondifferentiable potentials in the contact representation. In the literature, two different methods have been followed for the numerical solution process. The first consists of applying Newton's method to the saddle-point equations of the augmented Lagrangian [8] or, equivalently, of the perturbed Lagrangian [5, 6, 27]. The main drawback of this algorithm is that the size of the system increases because both displacements and tractions (or multipliers) remain together as independent unknown quantities. The second method consists of using Usawa's algorithm. This approach was chosen by Fremond [28-30] for contact problems without friction, and by Jean and Touzot $[9,23-25]$ and Franchomme et 
al. [31] for contact with friction. The current iteration involves two sets of predictor and corrector steps, one for unilateral contact and the other for friction. In the present approach, Usawa's algorithm is considered but the iteration is reduced to a single set of predictor and corrector steps. A similar algorithm using a projection step was presented by Giannakopoulos [32] and by Curnier and Alart [7] but starting from an approximation of the unilateral contact law by classical penalty techniques and of the friction law by a fictious elastoplastic-like law. This is an extension of the approach known in elastoplasticity as the return mapping method, which is actually a particular case of Moreau's catching-up algorithm [1, 3, 4].

Others numerical formulations, such as linear complementarity problems (LCP), have been introduced. Klarbring and Bjorkman [33] used a rigidplastic dry friction model that can be considered as a piecewise linearization of the implicit standard material model. Zhong and Sun [34] also used the LCP method but with a fictious elastoplastic-like model.

Other relevant features of contact problems, such as dual solutions by equilibrium and hybrid finite elements or accuracy problems due to geometrical modeling by finite elements, have been analyzed previously by De Saxce and Nguyen Dang Hung [35-38].

\section{IMPLICIT STANDARD MATERIALS}

In solid mechanics, a wide range of material behavior can be represented by the relation

$$
\mathbf{x}=\mathbf{f}(\mathbf{y})
$$

where $\mathbf{x}$ and $\mathbf{y}$ are, respectively, the generalized strain and stress vectors. These quantities may be understood in the context of instantaneous values, velocities, or finite increments. If $\mathrm{Eq} .1$ can be inverted,

$$
\mathbf{y}=\mathbf{g}(\mathbf{x})
$$

This definition is very broad, but generally gives little relevant information about the properties required for solving the boundary-value problem; i.e. the existence and uniqueness of solutions. These difficulties can be reflected in the numerical implementation of the solution procedure [1].

A more restricted range of material that leads to the good properties of the boundary-value problem is one of the so-called standard materials. The existence of potentials $V(\mathbf{x})$ and $W(\mathbf{y})$ is postulated. They are dual in Le- 
gendre's sense $[10-15,19,35,39]$; i.e.,

$$
V(\mathbf{x})+W(\mathbf{y})=\mathbf{x} \cdot \mathbf{y}
$$

This scalar relation defines the energetic law of the material. The potential $V$ represents a strain energy (or power) and $W$ represents a cornplementary energy. If $V$ and $W$ are differentiable, the material law in a vectorial form can be deduced from Eq. 3 as

$$
\mathbf{y}=\frac{\partial V(\mathbf{x})}{\partial \mathbf{x}}, \quad \mathbf{x}=\frac{\partial W(\mathbf{y})}{\partial \mathbf{y}}
$$

These equations are particular cases of Eqs. 1 and 2. For behavior such as plasticity, viscoplasticity, and contact, the concept of superpotential in the Moreau sense $[11,12]$ can be introduced, where $V(\mathbf{x})$ and $W(\mathbf{y})$ are assumed to be convex functions. Let $x^{\prime}$ and $y^{\prime}$ be any strain and stress vectors that are not related a priori by the material law. For all $\mathbf{x}^{\prime}$ and $\mathbf{y}^{\prime}$,

$$
V\left(\mathbf{x}^{\prime}\right)+W\left(\mathbf{y}^{\prime}\right) \geq \mathbf{x}^{\prime} \cdot \mathbf{y}^{\prime}
$$

where the equality is satisfied when the strain and stress vectors are related by the normality law of Eq. 4 . From Eqs. 3 and 5 ,

$$
\begin{array}{r}
V\left(\mathbf{x}^{\prime}\right)-V(\mathbf{x}) \geq \mathbf{y} \cdot\left(\mathbf{x}^{\prime}-\mathbf{x}\right) \\
W\left(\mathbf{y}^{\prime}\right)-W(\mathbf{y}) \geq \mathbf{x} \cdot\left(\mathbf{y}^{\prime}-\mathbf{y}\right)
\end{array}
$$

for all $\mathbf{x}^{\prime}$ and $\mathbf{y}^{\prime}$. In other words, $\mathbf{x}$ and $\mathbf{y}$ are linked by a law in a subdifferential form $[10-13,19,35,39]$, i.e.,

$$
\mathbf{y} \in \partial V(\mathbf{x}), \quad \mathbf{x} \in \partial W(\mathbf{y})
$$

Nevertheless, numerous behaviors are encountered in solid mechanics that do not belong to this useful family of standard materials. Some plasticity laws for metals [9], soils [1], and contact problems with friction [12, 14, $22,23]$ can be cited as examples.

It seems that a good generalization must preserve the notion of normality law in a suitable form and the convexity assumptions. A possible generalization consists of postulating the existence of a function $b(\mathbf{x}, \mathbf{y})$ that will be called bipotential such that the material law is

$$
b(\mathbf{x}, \mathbf{y})=\mathbf{x} \cdot \mathbf{y}
$$


If $b$ is differentiable, it can be deduced that

$$
\mathbf{y}=\frac{\partial b(\mathbf{x}, \mathbf{y})}{\partial \mathbf{x}}, \quad \mathbf{x}=\frac{\partial b(\mathbf{x}, \mathbf{y})}{\partial \mathbf{y}}
$$

The dependence of $\mathbf{y}$ on $\mathbf{x}$ is now implicit in the sense of the implicit function theorem, as opposed to the explicit dependence occurring in the law of Eq. 4 for standard materials. It may be noted that the standard materials correspond to a particular case of Eq. 8 for which the bipotential is separable; i.e.,

$$
b(\mathbf{x}, \mathbf{y})=V(\mathbf{x})+W(\mathbf{y})
$$

Later, the classical standard materials will be called the explicit standard materials, and materials with nonseparable bipotential will be called implicit standard materials.

In a more general way, it can be assumed that the bipotential is convex with respect to $\mathbf{x}$ when $\mathbf{y}$ remains constant, and convex with respect to $\mathbf{y}$ when $\mathbf{x}$ remains constant. For any $\mathbf{x}^{\prime}$ and $\mathbf{y}^{\prime}$ that are not related by the material law,

$$
b\left(\mathbf{x}^{\prime}, \mathbf{y}^{\prime}\right) \geq \mathbf{x}^{\prime} \cdot \mathbf{y}^{\prime}
$$

where equality occurs for $x^{\prime}$ and $\mathbf{y}^{\prime}$ related by the material law of Eq. 8 . From Eqs. 8 and 11, it can be deduced that

$$
\begin{aligned}
& b\left(\mathbf{x}^{\prime}, \mathbf{y}\right)-b(\mathbf{x}, \mathbf{y}) \geq \mathbf{y} \cdot\left(\mathbf{x}^{\prime}-\mathbf{x}\right) \\
& b\left(\mathbf{x}, \mathbf{y}^{\prime}\right)-b(\mathbf{x}, \mathbf{y}) \geq \mathbf{x} \cdot\left(\mathbf{y}^{\prime}-\mathbf{y}\right)
\end{aligned}
$$

for all $\mathbf{x}^{\prime}$ and $\mathbf{y}^{\prime}$. Then, $\mathbf{x}$ and $\mathbf{y}$ are linked by an implicit subdifferential law,

$$
\begin{aligned}
& \mathbf{y} \in \partial_{\mathbf{x}} b(\mathbf{x}, \mathbf{y}) \\
& \mathbf{x} \in \partial_{\mathbf{y}} b(\mathbf{x}, \mathbf{y})
\end{aligned}
$$

Attention is now focused on the first relation (Eq. 13a). It can be proved that the material belongs to the class defined by Eq. 1. This fact results from the convexity hypothesis of the bipotential. Indeed, the convex conjugate potential with respect to $\mathbf{x}$ can be defined as

$$
b_{1}^{*}(\mathbf{z}, \mathbf{y})=\sup _{\mathbf{x}}[\mathbf{z} \cdot \mathbf{x}-b(\mathbf{x}, \mathbf{y})]
$$


This potential will be called the left dual potential and $\mathbf{z}$, the left stresses. This implies that [39]

$$
\mathbf{z} \in \partial_{\mathbf{x}} b(\mathbf{x}, \mathbf{y}) \Leftrightarrow \mathbf{x} \in \partial_{\mathbf{z}} b_{1}^{*}(\mathbf{z}, \mathbf{y})
$$

If $\mathbf{y}$ is a solution of Eq. 13a,

$$
\mathbf{x} \in \partial_{\mathbf{z}} b_{i}^{*}(\mathbf{y}, \mathbf{y})
$$

The left dual potential depends on two stresses, $z$ and $y$. It can be said that $\mathbf{X}$ is obtained by subdifferentiating with respect to the left stresses $\mathbf{z}$ and taking the value $z=y$, without explicitly subdifferentiating with respect to y. In other words, the law of Eq. 16, a particular case of Eq. 1, defines the material as explicit nonstandard, as opposed to the implicit standard form (Eq. 13a). Hence, explicitly solving Eq. 13a has the drawback of losing the standard form of the law.

In a similar way, a right dual potential can be defined by

$$
b_{r}^{*}(\mathbf{x}, \mathbf{u})=\sup _{\mathbf{y}}[\mathbf{u} \cdot \mathbf{y}-b(\mathbf{x}, \mathbf{y})]
$$

The vector $\mathbf{u}$ denotes the so-called right strain.

If $\mathbf{x}$ is a solution of Eq. 13b,

$$
\mathbf{y} \in \partial_{\mathbf{u}} b_{r}^{*}(\mathbf{x}, \mathbf{x})
$$

\section{VARIATIONAL PRINCIPLES}

Let $\Omega$ be a structure with boundary $S$, subjected to imposed body forces f, imposed surface tractions $\bar{t}$ on part $S_{1}$ of $S$, and imposed displacements $\overline{\mathbf{u}}$ on part $S_{0}$ of $S$. On the remaining part $S_{2}=S-S_{0} \cup S_{1}$ of the boundary, contact may occur.

A displacement field is called kinematically admissible (KA) if the following compatibility conditions are fulfilled:

$$
\begin{aligned}
\varepsilon\left(\mathbf{u}^{k}\right) & =\operatorname{grad}_{s} \mathbf{u}^{k} \quad \text { in } \Omega \\
\mathbf{u}^{k} & =\overline{\mathbf{u}} \quad \text { on } S_{0}
\end{aligned}
$$

A stress field is said to be statically admissible (SA) if the following equilibrium equations are satisfied:

$$
\begin{aligned}
\operatorname{div} \boldsymbol{\sigma}^{s}+\mathbf{f} & =0 \quad \text { in } \Omega \\
\mathbf{t}\left(\boldsymbol{\sigma}^{s}\right) & =\boldsymbol{\sigma}^{s} \cdot \mathbf{n}=\overline{\mathbf{t}} \quad \text { on } S_{1}
\end{aligned}
$$


The aim of this section is to present a variational formulation for an implicit standard material behavior on the contact surface defined by a bipotential $b(\mathbf{u}, \mathbf{t})$ such that

$$
\mathbf{t} \in \partial_{-\mathbf{u}} b(-\mathbf{u}, \mathbf{t}), \quad-\mathbf{u} \in \partial_{\mathrm{t}} b(-\mathbf{u}, \mathbf{t})
$$

Unlike the general law of Eqs. 13, special sign conventions have been chosen to ensure compatibility with the classical traction definitions in solid mechanics [12]. For the sake of generality, an implicit standard behavior of the bodies is assumed by introducing a bipotential $\beta(\varepsilon, \sigma)$ such that

$$
\boldsymbol{\sigma} \in \partial_{\boldsymbol{\varepsilon}} \beta(\varepsilon, \boldsymbol{\sigma}), \quad \varepsilon \in \partial_{\boldsymbol{\sigma}} \beta(\varepsilon, \boldsymbol{\sigma})
$$

Hence, on this basis, the following new functional, called bifunctional, is defined:

$$
\begin{aligned}
B(\mathbf{u}, \boldsymbol{\sigma})= & \int_{\Omega} \beta(\boldsymbol{\varepsilon}(\mathbf{u}), \boldsymbol{\sigma}) d \Omega+\int_{S_{2}} b(\mathbf{u}, \mathbf{t}(\boldsymbol{\sigma})) d S \\
& -\int_{\Omega} \mathbf{f} \cdot \mathbf{u} d \Omega-\int_{S_{t}} \overline{\mathfrak{t}} \cdot \mathbf{u} d S-\int_{S_{\mathrm{o}}} \mathfrak{t}(\boldsymbol{\sigma}) \cdot \overline{\mathbf{u}} d S
\end{aligned}
$$

It may be noted that if it is assumed that no contact occurs $\left(S_{2}\right.$ is an empty set) and if the body material is explicit standard,

$$
\beta(\boldsymbol{\varepsilon}, \boldsymbol{\sigma})=V(\boldsymbol{\varepsilon})+W(\boldsymbol{\sigma})
$$

The bifunctional is reduced to the sum

$$
B(\mathbf{u}, \boldsymbol{\sigma})=\Phi(\mathbf{u})+\Pi(\boldsymbol{\sigma})
$$

of the total strain energy functional

$$
\Phi(\mathbf{u})=\int_{\Omega} V(\mathbf{\varepsilon}(\mathbf{u})) d \Omega-\int_{\Omega} \mathbf{f} \cdot \mathbf{u} d \Omega-\int_{S_{\mathfrak{l}}} \overline{\mathfrak{t}} \cdot \mathbf{u} d S
$$

and of the total complementary energy functional

$$
\Pi(\boldsymbol{\sigma})=\int_{\Omega} W(\boldsymbol{\sigma}) d \Omega-\int_{S_{0}} \mathfrak{t}(\boldsymbol{\sigma}) \cdot \overline{\mathbf{u}} d S
$$


The classical calculus of variations can now be extended to implicit standard materials. Because the bifunctional cannot be split further, the displacement and stress problems are coupled. Thus, an exact solution $(\mathbf{u}, \boldsymbol{\sigma})$ is simultaneously a solution of the following variational principles:

$$
\begin{aligned}
& \operatorname{Inf}_{\mathbf{u}^{k} K A} B\left(\mathbf{u}^{k}, \boldsymbol{\sigma}\right) \\
& \operatorname{lnf}_{\sigma^{s} S A} B\left(\mathbf{u}, \sigma^{5}\right)
\end{aligned}
$$

For example, consider the proof of the displacement principle. Due to Eqs. 19b, 21 , and 22 and the convexity inequality of Eq. 12a,

$$
\begin{aligned}
B\left(\mathbf{u}^{k}, \boldsymbol{\sigma}\right)-B(\mathbf{u}, \boldsymbol{\sigma}) \geq & \int_{\Omega} \boldsymbol{\sigma} \cdot\left(\boldsymbol{\varepsilon}\left(\mathbf{u}^{k}\right)-\boldsymbol{\varepsilon}(\mathbf{u})\right) d \Omega-\int_{\Omega} \mathbf{f} \cdot\left(\mathbf{u}^{k}-\mathbf{u}\right) d \Omega \\
& -\int_{S_{1}} \overline{\mathbf{t}} \cdot\left(\mathbf{u}^{k}-\mathbf{u}\right) d S-\int_{S_{2}} \mathbf{t}(\boldsymbol{\sigma}) \cdot\left(\mathbf{u}^{k}-\mathbf{u}\right) d S
\end{aligned}
$$

Since the exact solution $\sigma$ is statically admissible, the minimum principle results from equilibrium conditions of Eqs. 20 and Green's formula,

$$
B\left(\mathbf{u}^{k}, \boldsymbol{\sigma}\right) \geq B(\mathbf{u}, \boldsymbol{\sigma})
$$

In a similar way, the stress principle can be deduced from compatibility conditions of Eqs. 19. Consider the proof of the existence of a solution. For this, it is noted that the solution can be obtained by successive approximation and combination of the two principles. Let $\left(\mathbf{u}_{i}, \boldsymbol{\sigma}_{i}\right)$ be the approximative solution at iteration $i$. Let $\boldsymbol{\sigma}_{i+1}$ be a statically admissible stress field and $\mathbf{u}^{k}$, a kinematically admissible displacement field such that

$$
\boldsymbol{\varepsilon}\left(\mathbf{u}^{k}\right) \in \partial_{\boldsymbol{\sigma}} \boldsymbol{\beta}\left(\boldsymbol{\varepsilon}\left(\mathbf{u}_{i}\right), \mathbf{\sigma}_{i+1}\right), \quad-\mathbf{u}^{k} \in \partial_{\mathbf{t}} b\left(-\mathbf{u}_{i}, \mathbf{t}\left(\boldsymbol{\sigma}_{i+1}\right)\right)
$$

Then, because of the minimum principle (Eq. 28b),

$$
B\left(\mathbf{u}_{i}, \boldsymbol{\sigma}_{i}\right) \geq B\left(\mathbf{u}_{i}, \boldsymbol{\sigma}_{i+1}\right)
$$

Let $\mathbf{u}_{i+1}$ be a kinematically admissible displacement field and $\boldsymbol{\sigma}^{s}$, a statically admissible stress field such that

$$
\boldsymbol{\sigma}^{s} \in \partial_{\mathbf{e}} \beta\left(\varepsilon\left(\mathbf{u}_{i+1}\right), \boldsymbol{\sigma}_{i+1}\right), \quad \mathbf{t}\left(\boldsymbol{\sigma}^{s}\right) \in \partial_{-\mathbf{v}} b\left(-\mathbf{u}_{i+1}, \mathbf{t}\left(\boldsymbol{\sigma}_{i+1}\right)\right)
$$


From the minimum principle (Eq. 28a),

$$
B\left(\mathbf{u}_{i}, \boldsymbol{\sigma}_{i+1}\right) \geq B\left(\mathbf{u}_{i+1}, \boldsymbol{\sigma}_{i+1}\right)
$$

Hence, it is seen that a minimizing sequence of $B$ can be constructed.

Now, it is assumed that the couples belong to some reflexive Banach space $X$ with the norm $\|\cdot\|$. The existence of a solution of Eqs. 28 can be proved if the sequence $\left(u_{i}, \sigma_{i}\right)$ is bounded, by extracting a convergent subsequence $[12,39]$. The boundedness property can result from some special assumption or from a classical hypothesis of coercivity; i.e.,

$$
\lim _{\|(\mathbf{u}, \boldsymbol{\sigma})\|+\infty} B(\mathbf{u}, \boldsymbol{\sigma})=+\infty
$$

Let $\left(\mathbf{u}_{i^{\prime}}, \boldsymbol{\sigma}_{i^{\prime}}\right)$ be a bounded subsequence. Then

$$
\left(\mathbf{u}_{i^{\prime}}, \boldsymbol{\sigma}_{i^{\prime}}\right) \rightarrow(\mathbf{u}, \boldsymbol{\sigma}) \text { weakly in } X
$$

The method just described not only proves the existence of a solution, but gives also a practical algorithm to reach it. If the finite element method is used, this approximation algorithm requires the simultaneous definition of two kinds of meshes, the first involving displacement finite elements and the second involving equilibrium elements $[13,15]$.

A simpler method, using only displacement finite elements, is the following. The approximative solution $\left(\mathbf{u}_{i}, \boldsymbol{\sigma}_{i}\right)$ at iteration $i$ being known, the new kinematically admissible displacement approximation $\mathbf{u}_{i+1}$ is found by solving the following minimum problem:

$$
\operatorname{lnf}_{\mathbf{u}^{k} \mathrm{KA}} B\left(\mathbf{u}^{k}, \sigma_{i}\right)
$$

Thus, a stress field $\boldsymbol{\sigma}_{i+1}$ such that

$$
\begin{gathered}
\boldsymbol{\sigma}_{i+1} \in \partial_{\varepsilon} \beta\left(\boldsymbol{\varepsilon}\left(\mathbf{u}_{i+1}\right), \boldsymbol{\sigma}_{i}\right) \\
\mathbf{t}\left(\boldsymbol{\sigma}_{i+1}\right) \in \partial_{-\mathbf{u}} b\left(-\mathbf{u}_{i+1}, \mathbf{t}\left(\boldsymbol{\sigma}_{i}\right)\right)
\end{gathered}
$$

is statically admissible in a weak sense. In other words, it satisfies only the equilibrium equations pounderated by the displacement shape functions of the finite elements. 


\section{AUGMENTED LAGRANGIAN METHOD}

In order to avoid nondifferentiable potentials that occur in nonlinear mechanics, such as in contact problems, it is convenient to use the augmented Lagrangian method $[8,26]$ (see also the appendix of this paper). First, some classical results are recalled concerning this theory, applied to explicit standard materials. Let $r$ be a nonnegative real coefficient. Its value is chosen in a suitable range to ensure numerical convergence [26]. The convexity inequality (Eq. 6b) can be easily rewritten in the following way:

$$
r W\left(\mathbf{y}^{\prime}\right)-r W(\mathbf{y})+[\mathbf{y}-(\mathbf{y}+r \mathbf{x})] \cdot\left(\mathbf{y}^{\prime}-\mathbf{y}\right) \geq 0
$$

for all $\mathbf{y}^{\prime}$. Hence, $\mathbf{y}$ is the proximal of the so-called augmented stresses $(\mathbf{y}+r \mathbf{x})$ with respect to the function $r W$ :

$$
\mathbf{y}=\operatorname{prox}(\mathbf{y}+r \mathbf{x}, r w)
$$

The solution $y$ of this equation, equivalent to $\mathrm{Eq} .6 \mathrm{~b}$, can be reached using Usawa's algorithm. Let $\left(\mathbf{x}_{i}, \mathbf{y}_{i}\right)$ be the approximation at iteration $i$. The computation of $\mathbf{y}_{i+1}$ takes place in two steps, the predictor,

$$
\hat{\mathbf{y}}_{i+1}=\mathbf{y}_{i}+r \mathbf{x}_{i}
$$

and the corrector,

$$
\mathbf{y}_{i+1}=\operatorname{prox}\left(\hat{\mathbf{y}}_{i+1}, r W\right)
$$

In order to apply the algorithm to implicit standard materials, it is desired to extend the augmented Lagrangian method to the bipotential. The convexity inequality (Eq. 12b) is rewritten as

$$
r b\left(\mathbf{x}, \mathbf{y}^{\prime}\right)-r b(\mathbf{x}, \mathbf{y})+[\mathbf{y}-(\mathbf{y}+r \mathbf{x})] \cdot\left(\mathbf{y}^{\prime}-\mathbf{y}\right) \geq 0
$$

for all $y^{\prime}$. Hence, $y$ is the proximal of the augmented stress with respect to the function $r b(x, \cdot)$, where the variable argument is denoted by a dot and $\mathbf{x}$ remains constant; i.e.,

$$
\mathbf{y}=\operatorname{prox}(\mathbf{y}+r \mathbf{x}, r b(\mathbf{x}, \cdot))
$$


Then, Usawa's algorithm leads to an iterative process involving two steps: the predictor,

$$
\hat{\mathbf{y}}_{i+1}=\mathbf{y}_{i}+r \mathbf{x}_{i}
$$

and the corrector,

$$
\mathbf{y}_{i+1}=\operatorname{prox}\left(\hat{\mathbf{y}}_{i+1}, r b\left(\mathbf{x}_{i}, \cdot\right)\right)
$$

\section{CONSTITUTIVE LAW OF FRICTION WITH CONTACT}

For the sake of simplicity, consider contact between two bodies $\Omega_{A}$ and $\Omega_{B}$, one of which may be a rigid foundation. In the range of small displacements, it is a priori allowed to assume that possible contact may happen between points $x_{A}$ and $x_{B}$ of the two bodies. Consequently, the contact law can be written only in terms of the relative displacement $\mathbf{u}=\mathbf{u}_{A}-\mathbf{u}_{B}$ and the traction $t=t_{A}=-t_{B}$. With the approximation of small displacement, the unilateral contact condition is linearized as [35-38]

$$
u_{n}+h_{0} \geq 0
$$

where occurs, respectively, the normal unit vector and the initial gap,

$$
\mathbf{n}=\frac{\mathbf{x}_{A}-\mathbf{x}_{B}}{\left\|\mathbf{x}_{A}-\mathbf{x}_{B}\right\|}, \quad h_{0}=\left(\mathbf{x}_{A}-\mathbf{x}_{B}\right) \cdot \mathbf{n}
$$

and the decomposition of a vector $\mathbf{a}$ in normal and tangential parts is used; i.e.,

$$
a_{n}=\mathbf{a} \cdot \mathbf{n}, \quad \mathbf{a}_{\mathbf{i}}=\mathbf{a}-a_{n} \mathbf{n}
$$

Besides, Coulomb's dry friction criterion is used; i.e.,

$$
\left\|\mathbf{t}_{\boldsymbol{t}}\right\| \leq \mu t_{n}
$$

With the usual notation of an indicator function $\Psi_{\mathbf{R}+}$ (see the appendix), the constitutive law of contact can be thus written as

$$
t_{n} \in-\partial \Psi_{\mathbf{R}+}\left(u_{n}+h_{0}\right), \quad \mathbf{t}_{t} \in-\mu t_{n} \partial_{\dot{\mathbf{u}}_{t}}\left(\left\|\dot{\mathbf{u}}_{\|}\right\|\right)
$$

where an overdot denotes the time derivative. 
In view of numerical applications, the implicit standard material law of contact is directly presented in the incremental form

$$
\Delta \mathbf{t} \in \partial_{-\Delta \mathbf{u}} b(-\Delta \mathbf{u}, \Delta \mathbf{t}), \quad-\Delta \mathbf{u} \in \partial_{\Delta \mathbf{t}} b(-\Delta \mathbf{u}, \Delta \mathbf{t})
$$

Let $\Delta K_{\mu}$ be the convex friction set defined by

$$
\Delta K_{\mu}=\left\{\left(\Delta t_{n}, \Delta \mathbf{t}_{t}\right) \quad \text { such that } \quad\left\|\mathbf{t}_{n 0}+\Delta \mathbf{t}_{r}\right\| \leq \mu\left(t_{n 0}+\Delta t_{n}\right)\right\}
$$

where the values at the starting point of the step are noted with a subscript 0 . Contact with friction can be represented by the following bipotential:

$$
\begin{aligned}
b\left(-\Delta u_{n},-\Delta \mathbf{u}_{t}, \Delta t_{n}, \Delta \mathbf{t}_{t}\right)= & t_{n 0} \Delta u_{n}+\mathbf{t}_{t 0} \cdot \Delta \mathbf{u}_{t}+\Delta t_{n}\left(u_{n 0}+h_{0}\right) \\
& +\mu\left(t_{n 0}+\Delta t_{n}\right)\left\|\Delta \mathbf{u}_{t}\right\| \\
& +\Psi_{\Delta K_{\mu}}\left(\Delta t_{n}, \Delta \mathbf{t}_{t}\right) \\
& +\Psi_{\mathbf{R}+}\left(\Delta u_{n}+u_{n 0}+h_{0}\right)
\end{aligned}
$$

Hence, the Iaw of Eq. 13a gives

$$
\begin{aligned}
& \Delta t_{n} \in-\left(t_{n 0}+\partial \Psi_{\mathbf{R}+}\left(\Delta u_{n}+u_{n 0}+h_{0}\right)\right) \\
& \Delta \mathbf{t}_{t} \in-\left(\mathbf{t}_{t 0}+\mu\left(t_{n 0}+\Delta t_{n}\right) \partial_{\Delta u_{i}}\left(\left\|\Delta \mathbf{u}_{r}\right\|\right)\right)
\end{aligned}
$$

Equation 55a represents the unilateral contact law and Eq. 55b is Coulomb's criterion in subdifferential form. These equations may be directly deduced from the multivalued differential equations (Eqs. 51) by applying the implicit method [1-4]. It is well known that the implicit method, although more complicated, always gives better results concerning convergence and numerical stability than the explicit method [1-4]. The implicit method has been discussed by Moreau [3] and is known as the catching-up algorithm.

On the other hand, introducing some subnormal to Coulomb's cone

$$
\left(\Delta v_{n}, \Delta \mathbf{v}_{t}\right) \in \partial \Psi_{\Delta K_{\mu}}\left(\Delta t_{n}, \Delta \mathbf{t}_{t}\right)
$$

Eq. $13 b$ yields

$$
\Delta u_{n}=-\left(u_{n 0}+h_{0}+\mu\left\|\Delta \mathbf{u}_{t}\right\|+\Delta v_{n}\right), \quad \Delta \mathbf{u}_{t}=-\Delta \mathbf{v}_{t}
$$

As Eq. 56 leads to

$$
\mu\left\|\Delta \mathbf{v}_{n}\right\|+\Delta v_{n} \leq 0
$$


it may be seen that Eq. 57 is reduced to the unilateral contact condition:

$$
\Delta u_{n}+u_{n 0}+h_{0} \geq 0
$$

Except for the cone vertex $\left(t_{m 0}+\Delta t_{n}=0\right)$, equality is reached in Eqs. 58 and 59. Hence, the law of Eq. 57 is equivalent to the classical sliding law $[12,14]$; i.e.,

$$
\Delta \mathbf{u}_{t}=-\Delta \lambda \frac{\mathbf{t}_{00}+\Delta \mathbf{t}_{t}}{\left\|\mathbf{t}_{10}+\Delta \mathbf{t}_{t}\right\|}, \quad \Delta \lambda \geq 0, \quad \Delta u_{n}=-\left(u_{m 0}+h_{0}\right)
$$

Another simple geometrical interpretation of Eq. 57 can be obtained by noting that when sliding occurs (equality in Eq. 58), Eqs. 56 and 57 imply that the vector $\left(-\mu\left\|\Delta \mathbf{u}_{\|}\right\|, \Delta \mathbf{u}_{t}\right)$ is a normal to Coulomb's cone; i.e.,

$$
\left(-\mu\left\|\Delta \mathbf{u}_{t}\right\|, \Delta \mathbf{u}_{t}\right) \in \partial \Psi_{\Delta K_{\mu}}\left(\Delta t_{n}, \Delta \mathbf{t}_{t}\right)
$$

as is represented in Fig. 1.

The model of implicit standard material provides a simple and powerful tool to represent the complex behavior of contact with friction. This model can be compared with the classical model of explicit laws. By Legendre's transform of the bipotential of Eq. 54 with respect to the displacement, the

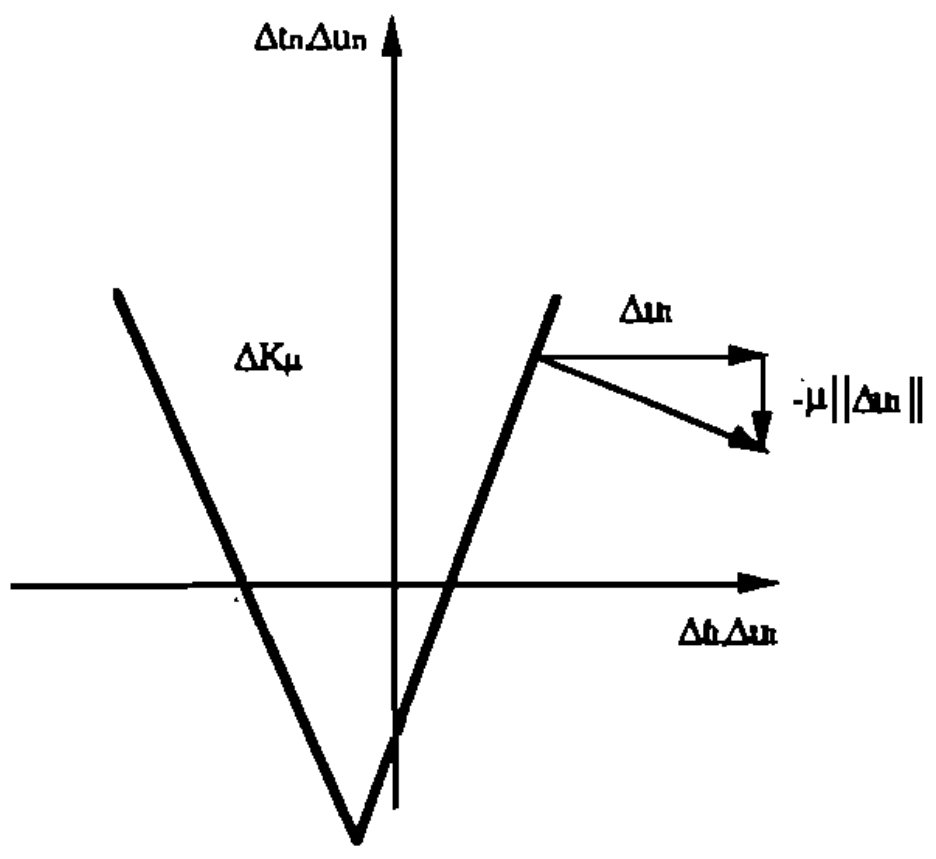

Fig. 1 Sliding. 
left dual potential of Eq. 14 is obtained:

$$
\begin{aligned}
b_{1}^{*}\left(\Delta \tilde{t}_{n}, \Delta \overline{\mathrm{t}}_{t}, \Delta t_{n}, \Delta \mathbf{t}_{t}\right)= & \left(t_{n 0}+\Delta \bar{t}_{n}-\Delta t_{n}\right)\left(u_{n 0}+h_{0}\right)+\Psi_{\mathbf{R}+}\left(t_{n 0}+\Delta \bar{t}_{n}\right) \\
& +\Psi_{\Delta K_{\mu}}\left(\Delta \bar{t}_{n}, \Delta \overline{\mathbf{t}}_{t}\right)-\Psi_{\Delta K_{\mu}}\left(\Delta t_{n}, \Delta \mathbf{t}_{t}\right)
\end{aligned}
$$

where $\Delta \overline{\mathbf{t}}$ denotes some left traction vector, dual to $-\Delta \mathbf{u}$. Owing to Eq. 16, the explicit form of the law is

$$
\Delta u_{n}+u_{n 0}+h_{0} \in-\partial \Psi_{\mathbf{R}+}\left(t_{n 0}+\Delta t_{n}\right), \quad \Delta \mathbf{u}_{t} \in-\partial_{\Delta 1_{i}} \Psi_{\Delta k_{\mu}}\left(\Delta t_{n}, \Delta \mathbf{t}_{t}\right)
$$

Obviously, these equations are the inverse relations of Eqs. 55, but they are not as easy to use for numerical analysis.

\section{VARIATIONAL PRINCIPLES FOR CONTACT WITH FRICTION}

As shown in Section III, the boundary-value problem of contact with friction is governed by the variational principles of Eqs. 28. In order to prove the existence of solutions, coercivity of the bifunctional must be checked. This property may be assumed for the body material. For example, in numerical applications, the bodies are elastic, with the explicit standard model

$$
\beta(\boldsymbol{\epsilon}, \boldsymbol{\sigma})=V(\boldsymbol{\epsilon})+W(\boldsymbol{\sigma}), \quad V(\boldsymbol{\epsilon})=\frac{1}{2} \boldsymbol{\epsilon} \cdot D \boldsymbol{\varepsilon}, \quad W(\boldsymbol{\sigma})=\frac{1}{2} \boldsymbol{\sigma} \cdot \mathbf{D}^{-1} \boldsymbol{\sigma}
$$

Then, because the elastic stiffness matrix $D$ is positive-definite, the potentials $V$ and $W$, and consequently the bipotential $\beta$, are coercive functions.

Attention is next focused on the contact bipotential of Eq. 54. It may be assumed that for the initial values of the step (i.e., at the end of the previous step), the unilateral contact inequalities are fulfilled:

$$
\begin{aligned}
u_{n 0}+h_{0} & \geq 0 \\
t_{n 0} & \geq 0
\end{aligned}
$$

Because of the unilateral contact conditions of Eq. 59, $\Delta u_{n}$ is bounded for negative values. When the contact initially occurs on some part of $S_{2}$ with nonvanishing area, the inequality of Eq. $65 \mathrm{~b}$ is strictly satisfied and the bipotential increases to an infinite value when $\Delta u_{n} \rightarrow+\infty$. Elsewhere, some special hypotheses concerning the applied loads must be introduced in order to satisfy coercivity as proved by Fremond [28-30]. 
Because of Coulomb's condition (Eq. .53), it may be assumed that

$$
t_{n 0}+\Delta t_{n} \geq 0
$$

Thus, $\Delta t_{n}$ is bounded for negative values. When the initial gap does not vanish on some part of $S_{2}$ with nonvanishing area, the inequality of $\mathrm{Eq} .65 \mathrm{a}$ is strictly satisfied and the bipotential increases to infinity when $\Delta t_{n} \rightarrow+\infty$. Elsewhere, a special hypothesis is needed, as previously.

Due to Eq. 66, coercivity of the bipotential is proved when $\left\|\Delta \mathbf{u}_{n}\right\| \rightarrow+\infty$. Finally, $\Delta t$, is bounded by Coulomb's condition (Eq. 53).

For the sake of simplicity, analysis is restricted to contact between elastic bodies. When using pure displacement finite elements, only the principle of Eq. 28a need be considered. Introducing the incremental total strain energy

$$
\Delta \Phi(\Delta \mathbf{u})=\int_{\Omega} V(\Delta \mathbf{\varepsilon}(\Delta \mathbf{u})) d \Omega-\int_{\Omega} \Delta \mathbf{f} \cdot \Delta \mathbf{u} d \Omega-\int_{S_{1}} \Delta \overline{\mathbf{t}} \cdot \Delta \mathbf{u} d S
$$

the implicit standard material model leads to the following variational principle:

$$
\begin{aligned}
\operatorname{lnf} \Delta \Phi_{b}(\Delta \mathbf{u})= & \Delta \Phi(\Delta \mathbf{u}) \\
& +\int_{S_{2}}\left(t_{n 0} \Delta u_{n}+\mathbf{t}_{n 0} \cdot \Delta \mathbf{u}_{t}+\mu\left(t_{n 0}+\Delta t_{n}\right)\left\|\Delta \mathbf{u}_{t}\right\|\right) d S
\end{aligned}
$$

for KA $\Delta \mathbf{u}$, subject to

$$
\Delta u_{n}+u_{n 0}+h_{0} \geq 0 \text { on } S_{2}
$$

This variational principle was proposed by various authors [40, 41].

In opposition to classical variational principles, this one must be solved by an iterative algorithm. The normal contact pressure increment $\Delta t_{n}$ can be updated at each iteration using Eq. 38b. The iterations are required by the implicit standard nature of the material laws. The main advantage of this approach is that unilateral contact and friction are coupled in a single displacement variational principle.

In the classical approach as presented by Duvaut and Lions [14] or Panagiatopoulos [12, 22], the algorithm is also iterative, but unilateral contact and friction are not coupled: there are two displacement variational principles. For the first $[12,14,22,38]$, the tangential traction increments $\Delta t$, are 
assumed constant and only the unilateral contact problem is considered:

$$
\operatorname{lnf} \Delta \Phi_{c}(\Delta \mathbf{u})=\Delta \Phi(\Delta \mathbf{u})+\int_{S_{2}}\left(t_{n 0} \Delta u_{n}-\Delta \mathbf{t}_{t} \cdot \Delta \mathbf{u}_{t}\right) d S
$$

for KA $\Delta \mathbf{u}$ subjected to

$$
\Delta u_{n}+u_{n 0}+h_{0} \geq 0 \quad \text { on } S_{2}
$$

For the second, the normal contact pressure increment $\Delta t_{n}$ remains constant and the variational problem is

$$
\begin{aligned}
\operatorname{Inf} \Delta \Phi_{f}(\Delta \mathbf{u})= & \Delta \Phi(\Delta \mathbf{u}) \\
& +\int_{S_{2}}\left(\mu\left(t_{n 0}+\Delta t_{n}\right)\left\|\Delta \mathbf{u}_{r}\right\|+\mathbf{t}_{f 0} \cdot \Delta \mathbf{u}_{t}-\Delta t_{n} \Delta u_{n}\right) d S
\end{aligned}
$$

for KA $\Delta \mathbf{u}$. The two principles are alternately solved and the traction increments updated.

When using equilibrium finite elements, the dual principle of Eq. $28 \mathrm{~b}$ can be introduced. Let the incremental total complementary energy be

$$
\Delta \Pi(\Delta \boldsymbol{\sigma})=\int_{\Omega} W(\Delta \boldsymbol{\sigma}) d \Omega-\int_{S_{0}} \Delta \mathbf{t}(\Delta \boldsymbol{\sigma}) \cdot \Delta \overline{\mathbf{u}} d S
$$

Taking account of Eq. 54, the principle of stress variation for the implicit standard model is given by

$$
\operatorname{Inf} \Delta \Pi_{b}(\Delta \boldsymbol{\sigma})=\Delta \Pi(\Delta \boldsymbol{\sigma})+\int_{S_{2}}\left(\Delta t_{n}\left(u_{n 0}+h_{0}\right)+\mu\left(t_{n 0}+\Delta t_{n}\right)\left\|\Delta \mathbf{u}_{n}\right\|\right) d S
$$

for SA $\Delta \boldsymbol{\sigma}$ subjected to

$$
\left\|\mathbf{t}_{t 0}+\Delta \mathbf{t}_{t}\right\| \leq \mu\left(t_{n 0}+\Delta t_{n}\right) \quad \text { on } S_{2}
$$

\section{VARIATIONAL INEQUALITIES OF CONTACT}

In this approach, the traction field on $S_{2}$ is expressed with respect to the displacement field on $S_{2}$ by solving equilibrium equations, linear if the bod- 
ies are elastic and linearized if they are inelastic. The philosophy of this approach is well explained by Suquet [18] and is called global GSM theory.

The existence of a strongly monotonic affine operator and of its inverse is assumed:

$$
\begin{aligned}
E & : \Delta \mathbf{t} \rightarrow \Delta \hat{\mathbf{u}}(\Delta \mathbf{t}) \\
E^{-1}: \Delta \mathbf{u} & \rightarrow \Delta \hat{\mathbf{t}}(\Delta \mathbf{u})
\end{aligned}
$$

Using the convexity inequality of Eq. 12b applied to the bipotential of Eq. 54 and Eq. 73a, it may be seen that the exact field $\Delta t$ is a solution of the following variational inequality:

Find $\Delta \mathrm{t} \in \Delta K_{\mu}$ such that

$$
\begin{aligned}
& \int_{S_{2}}\left(\Delta \hat{u}_{n}\left(\Delta t_{n}, \Delta \mathbf{t}_{t}\right)+u_{n 0}+h_{0}+\mu\left\|\Delta \hat{\mathbf{u}}_{t}\left(\Delta t_{n}, \Delta \mathbf{t}_{t}\right)\right\|\right) \cdot\left(\Delta t_{n}^{*}-\Delta t_{n}\right) d S \\
& \quad+\int_{S_{2}} \Delta \hat{\mathbf{u}}_{t}\left(\Delta t_{n}, \Delta \mathbf{t}_{t}\right) \cdot\left(\Delta \mathbf{t}_{t}^{*}-\Delta \mathbf{t}_{t}\right) d S \geq 0
\end{aligned}
$$

for all $\Delta \mathbf{t}^{*} \in \Delta K_{\mu}$.

This new inequality is the formulation associated with the implicit standard material law of Eq. 57.

The dual formulation is obtained by considering Eqs. 12a and 73b. Hence, the exact field $\Delta \mathbf{u}$ is a solution of the following variational inequalities:

Find $\Delta \mathrm{u}$ such that $\Delta u_{n}+u_{n 0}+h_{0} \geq 0$ and

$$
\begin{aligned}
& \int_{S_{2}}\left(\mathbf{t}_{t 0}+\Delta \hat{t_{t}}\left(\Delta u_{n}, \Delta \mathbf{u}_{t}\right)\right) \cdot\left(\Delta \mathbf{u}_{t}^{*}-\Delta \mathbf{u}_{t}\right) d S \\
& \quad+\int_{S_{2}} \mu\left(t_{n 0}+\Delta \hat{t_{n}}\left(\Delta u_{n}, \Delta \mathbf{u}_{t}\right)\right)\left(\left\|\Delta \mathbf{u}_{t}^{*}\right\|-\left\|\Delta \mathbf{u}_{t}\right\|\right) d S \geq 0
\end{aligned}
$$

for all $\Delta \mathbf{u}_{t}^{*}$ and

$$
\int_{S_{2}}\left(t_{n 0}+\Delta \hat{t}_{n}\left(\Delta u_{n}, \Delta \mathbf{u}_{t}\right)\right)\left(\Delta u_{n}^{*}-\Delta u_{n}\right) d S \geq 0
$$

for all $\Delta u_{n}^{*}$ such that $\Delta u_{n}^{*}+u_{n 0}+h_{0} \geq 0$. 
This system of inequalities corresponds to the implicit standard material law in the dual form of Eqs. 55. After piecewise linearization, this system is equivalent to the linear complementarity problem stated by Klarbring and Bjorkman [33].

Another approach consists of considering the explicit nonstandard formulation based on the left dual potential of Eq. 62 . Introducing the hyperball

$$
\Delta K_{\mu}\left(\Delta t_{n}\right)=\left\{\Delta \mathbf{t}_{t} \text { such that }\left\|\mathfrak{t}_{t 0}+\Delta \mathbf{t}_{n}\right\| \leq \mu\left(t_{n 0}+\Delta t_{n}\right)\right\}
$$

and owing to Eqs. 17 and $73 a$, it may be seen that the exact traction field $\Delta \mathrm{t}$ such that

$$
t_{n 0}+\Delta t_{n} \geq 0, \quad \Delta \mathrm{t}_{t} \in \Delta K_{\mu}\left(\Delta t_{n}\right)
$$

is a solution of the following system of variational inequalities:

Find $\Delta t_{n}$ such that $t_{n 0}+\Delta t_{n} \geq 0$ and

$$
\int_{S_{2}}\left(\Delta \hat{u}_{n}\left(\Delta t_{n}, \Delta \mathbf{t}_{t}\right)+u_{n 0}+h_{0}\left(\Delta t_{n}^{*}-\Delta t_{n}\right) d S \geq 0\right.
$$

for all $\Delta t_{n}^{*}$ such that $t_{n 0}+\Delta t_{n}^{*} \geq 0$.

Find $\Delta \mathrm{t}_{t} \in \Delta K_{\mu}\left(\Delta t_{n}\right)$ such that

$$
\int_{S_{2}} \Delta \hat{\mathbf{u}}_{t}\left(\Delta t_{n}, \Delta \mathbf{t}_{t}\right) \cdot\left(\Delta \mathbf{t}_{t}^{*}-\Delta \mathbf{t}_{t}\right) d S \geq 0
$$

for all $\Delta \mathrm{t}_{t}^{*} \in \Delta K_{\mu}\left(\Delta t_{n}\right)$.

These two variational inequalities must be solved alternately, as the two variational principles of Eqs. 69 and 70, which result from the explicit nonstandard nature of the formulation. The inequality formulation of Eq. 63 was presented by Jean [23].

\section{USAWA'S ALGORITHM FOR CONTACT PROBLEMS}

The global GSM approach is again considered, but instead of the inequality formulation, the augmented Lagrangian method is used. For the implicit standard material, Eq. 44 applied to the bipotential of Eq. 54 leads 
to the following equation:

$$
\Delta \mathbf{t}=\operatorname{proj}\left(\left(\Delta t_{n}-r\left(\Delta u_{n}+u_{n 0}+h_{0}+\mu\left\|\Delta \mathbf{u}_{t}\right\|\right), \Delta \mathbf{t}_{t}-r \Delta \mathbf{u}_{t}\right), \Delta K_{\mu}\right)
$$

This equation corresponds to the contact law in the implicit form of Eq. 57. Usawa's algorithm applied to Eq. 79 consists of an iterative process involving the predictor

$$
\begin{aligned}
& \Delta \tau_{n}^{i+1}=\Delta t_{n}^{i}-r\left(\Delta \hat{u}_{n}\left(\Delta t_{n}^{i}, \Delta \mathbf{t}_{t}^{i}\right)+u_{n 0}+h_{0}+\mu\left\|\Delta \hat{\mathbf{u}}_{t}\left(\Delta t_{n}^{i}, \Delta \mathbf{t}_{t}^{i}\right)\right\|\right) \\
& \Delta \tau_{t}^{i+1}=\Delta \mathbf{t}_{t}^{i}-r \Delta \hat{\mathbf{u}}_{t}\left(\Delta t_{n}^{i}, \Delta \mathbf{t}_{t}^{i}\right)
\end{aligned}
$$

and the corrector

$$
\Delta \mathrm{t}^{i+1}=\operatorname{proj}\left(\Delta \boldsymbol{\tau}^{i+1}, \Delta K_{\mu}\right)
$$

The corrector step is graphically represented in Fig. 2 for three different contact statuses, denoting by $\Delta K_{\mu}^{*}$ the dual cone of $\Delta K_{\mu}$ defined by Eq. 58 . In this approach, unilateral contact and friction are coupled.

The new approach may be compared with that developed by Jean and Touzot [9, 23-25] and by Alart [8], which requires two equations instead of the single equation of Eq. 79. The exact field $\Delta t$ is a solution of the following system:

$$
\begin{aligned}
\Delta t_{n} & =\operatorname{proj}\left(\Delta t_{n}-r\left(\Delta u_{n}+u_{n 0}+h_{0}\right),\left[-t_{n 0},+\infty\right]\right) \\
\Delta \mathbf{t}_{t} & =\operatorname{proj}\left(\Delta \mathbf{t}_{t}-r \Delta \mathbf{u}_{t}, \Delta K_{\mu}\left(\Delta t_{n}\right)\right)
\end{aligned}
$$

These equations correspond to contact in the explicit form of Eq. 63 .

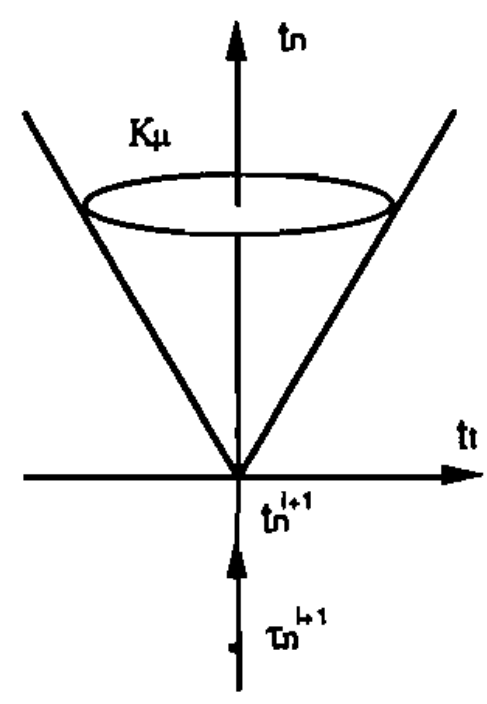

(a)

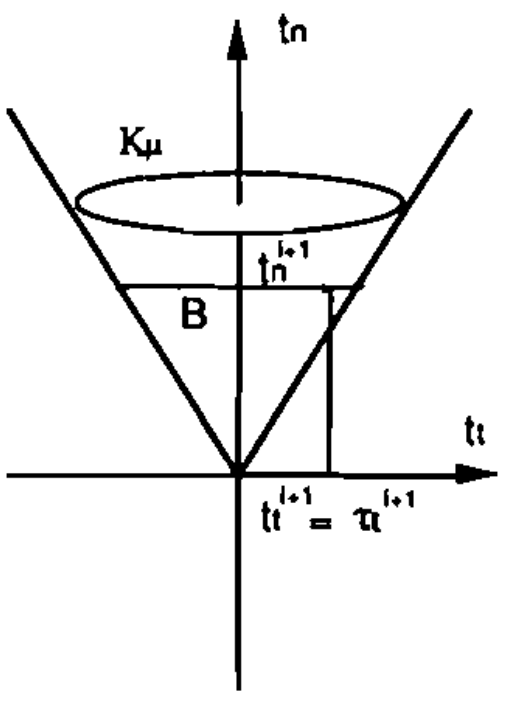

(b)

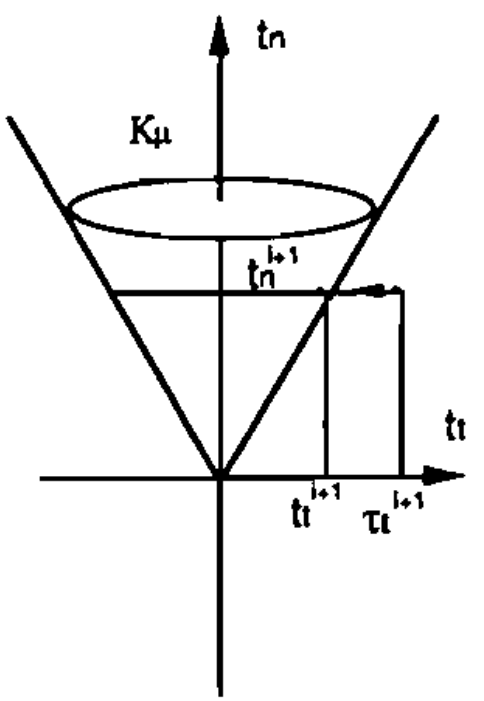

(c)

Fig. 2 Implicit standard material approach $\left(K_{j}^{*}\right.$ : dual cone of $\left.K_{\mu}\right)$ : (a) no contact; (b) adhesive friction; (c) sliding. 


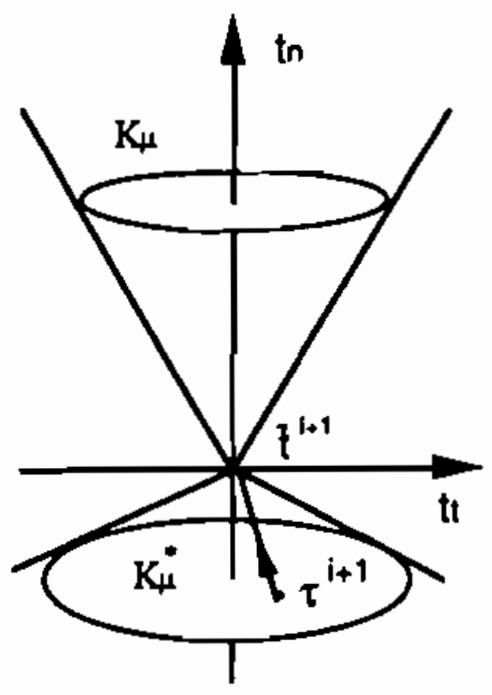

(a)

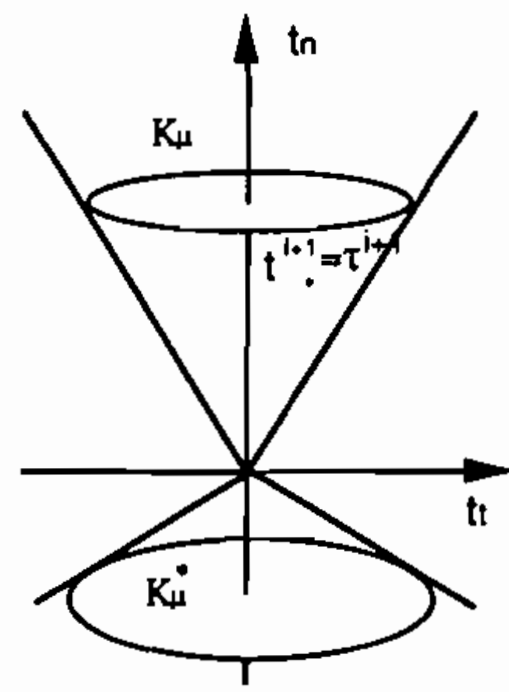

(b)

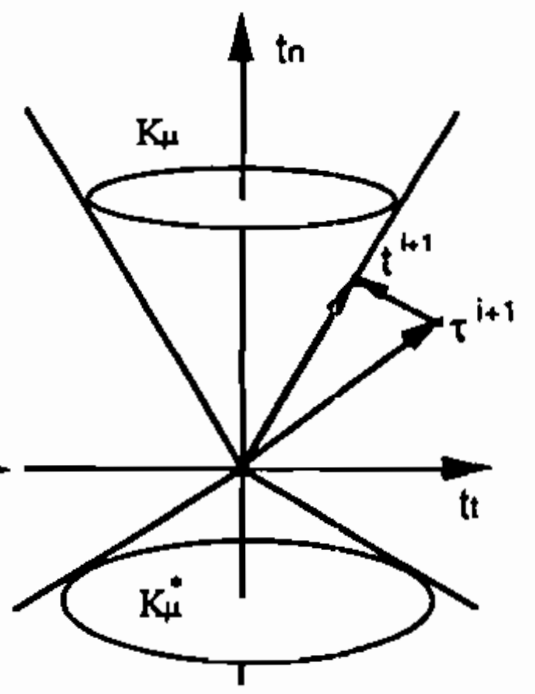

(c)

Fig. 3 Explicit material approach: (a) no contact; (b) adhesive friction; (c) sliding.

Following Jean $[9,23-25]$, Usawa's algorithm, applied to the unilateral contact equation of Eq. 82, leads to the predictor

$$
\Delta \tau_{n}^{i+1}=\Delta t_{n}^{i}-r\left(\Delta \hat{u}_{n}\left(\Delta t_{n}^{i}, \Delta \mathbf{t}_{i}^{i}\right)+u_{n 0}+h_{0}\right)
$$

and the corrector

$$
\Delta t_{n}^{i+1}=\operatorname{proj}\left(\Delta \tau_{n}^{i+1},\left[-t_{n 0},+\infty\right]\right)
$$

Applied to the friction equation (Eq. 83), this leads to the predictor

$$
\Delta \tau_{t}^{i+1}=\Delta \mathbf{t}_{t}^{i}-r \Delta \hat{\mathbf{u}}_{t}\left(\Delta t_{n}^{i+1}, \Delta \mathbf{t}_{t}^{i}\right)
$$

and the corrector

$$
\Delta \mathrm{t}_{t}^{i+1}=\operatorname{proj}\left(\Delta \tau_{t}^{i+1}, \Delta K_{\mu}\left(\Delta t_{n}^{i+1}\right)\right)
$$

The corrector step of Eq. 85 is graphically represented in Fig. 3(a) when contact is not predicted, and the corrector step of Eq. 87 is represented in Figs. 3(b) and 3(c).

\section{CONCLUSION}

The success of the standard material approach follows from the possibility of associating variational functionals to deduce good properties for the boundary-value problems. Surface friction laws are good examples of ma- 
terial behaviors that exhibit nonassociated flow rules. In order to avoid this undesirable lack of normality and consequently to extend the classical variational calculus to nonstandard materials, it is shown to be necessary to abandon the explicit form of the constitutive law. This idea leads to the introduction of the concept of a bipotential that generalizes the classical potentials of strain energy and complementary energy. The new formulation allows the unilateral contact and the friction law to be expressed as a single variational inequality. This variational inequality may be solved by using an adaptation of Usawa's algorithm, which requires only a single projection step to update the tractions instead of two steps, as required in some other algorithms.

Displacements are updated by computing a linear elastic response. This can be performed using classical finite element analysis. The details of the discretization procedure and the numerical results are reported in another paper.

\section{ACKNOWLEDGMENT}

The first author gratefully acknowledges the CNRS for support during his stay at the University of Compiegne, where this work was performed.

\section{APPENDIX}

Let $Y$ be a locally convex separated topological vector space and $Y^{\prime}$ its dual. The duality is denoted $\left\langle\mathbf{x}, \mathbf{y}^{\prime}\right\rangle$, for $\mathbf{x} \in Y$ and $\mathbf{y}^{\prime} \in Y^{\prime}$. Let $F$ be a convex function defined on $Y$, with values in $\overline{\mathbf{R}}=[-\infty,+\infty]$. The vector $\mathbf{y}^{\prime}$ is a subgradient of $F$ at the point $\mathbf{x}$ if $\mathbf{y}^{\prime}$ is the slope of an affine minorant of $F$ exact at the point $\mathbf{x}$. The set of subgradient of $F$ at $\mathbf{x}$ is the subderivative

$$
\partial F(\mathbf{x})=\left\{\mathbf{y}^{\prime} \in Y^{\prime} \text { such that } \forall \mathbf{u} \in Y, F(\mathbf{u})-F(\mathbf{x}) \geq\left\langle\mathbf{y}^{\prime}, \mathbf{u}-\mathbf{x}\right)\right\}
$$

If $F$ is differentiable, then $\partial F(\mathbf{x})=\left\{F^{\prime}(\mathbf{x})\right\}$.

Let $K \subset Y$ be a closed convex set. The indicator function of the convex set $K$ is denoted $\Psi_{K}(\mathbf{x})$ and is defined by

$$
\begin{aligned}
& \Psi_{K}(\mathbf{x})=0 \quad \text { if } \mathbf{x} \in K \\
& \Psi_{K}(\mathbf{x})=+\infty \quad \text { if } \mathbf{x} \notin K
\end{aligned}
$$

Then, for $\mathbf{x} \in K, \partial \Psi_{K}(\mathbf{x})=\left\{\mathbf{y}^{\prime} \in Y^{\prime}\right.$ such that $\left.\forall \mathbf{u} \in K,\left\langle\mathbf{y}^{\prime}, \mathbf{u}-\mathbf{x}\right\rangle \leq 0\right\}$. 
If $\mathbf{x}$ belongs to the interior of $K$, then $\partial \Psi_{K}(\mathbf{x})=\{0\}$. If $\mathbf{x}$ is on the boundary of $K, \partial \Psi_{K}(\mathbf{x})$ is the outward normal cone of $K$ at the point $\mathbf{x}$.

The conjugate functional $F^{*}$ is defined on $Y^{\prime}$ by the Legendre transform

$$
F^{*}\left(\mathbf{y}^{\prime}\right)=\sup _{\mathbf{u} \in Y}\left(\left\langle\mathbf{y}^{\prime}, \mathbf{u}\right\rangle-F(\mathbf{u})\right)
$$

so that $F^{*}\left(\mathbf{y}^{\prime}\right)=\left\langle\mathbf{y}^{\prime}, \mathbf{x}\right\rangle-F(\mathbf{x})$ when $\mathbf{y}^{\prime} \in \partial F(\mathbf{x})$. Furthermore, $\mathbf{x} \in \partial F^{*}\left(\mathbf{y}^{\prime}\right)$.

Let $H$ be a Hilbert space for the inner product $(\mathbf{x} \mid \mathbf{y})$ and the norm $\|\mathbf{x}\|=$ $\sqrt{(\mathbf{x} \mid \mathbf{x})}$. The duality mapping $\mathrm{F}$ from $H$ to its dual $H^{\prime}$ is defined by

$$
\langle\mathbf{F x}, \mathbf{y}\rangle=(\mathbf{x} \mid \mathbf{y})
$$

Let $f$ be a convex function on $H$ with values in $\overline{\mathbf{R}}=[-\infty,+\infty]$ and $\mathrm{x}$ be given in $H$. The solution $\mathbf{y}$ of the following minimization problem

$$
\inf _{\mathbf{u} \in H}\left(f(\mathbf{u})+\frac{\mathbf{l}}{2}\|\mathbf{x}-\mathbf{u}\|^{2}\right)
$$

is called the proximal point of $\mathbf{x}$ with respect to the function $f$ and is denoted

$$
\mathbf{y}=\operatorname{prox}(\mathbf{x}, f)
$$

Hence, the proximal point satisfies

$$
\mathbf{F}(\mathbf{x}-\mathbf{y}) \in \partial f(\mathbf{y})
$$

or, equivalently,

$$
f\left(\mathbf{y}^{*}\right)-f(\mathbf{y})+\left(\mathbf{y}-\mathbf{x} \mid \mathbf{y}^{*}-\mathbf{y}\right) \geq 0
$$

for all $\mathbf{y}^{*}$. The proximation mapping introduced by Moreau is defined by

$$
\operatorname{prox}_{f}: H \rightarrow H: \mathbf{x} \rightarrow \operatorname{prox}(\mathbf{x}, f)
$$

Let $K \subset H$ be a closed convex set. Then, the proximal point with respect to the indicator function $\Psi_{K}$ is a point $\mathbf{y} \in K$ such that for all $\mathbf{y}^{*} \in K$,

$$
\left(\mathbf{y}-\mathbf{x} \mid \mathbf{y}^{*}-\mathbf{y}\right) \geq 0
$$

Then, the proximal point is the projection of $\mathbf{x}$ to the set $K$ :

$$
\mathbf{y}=\operatorname{prox}\left(\mathbf{x}, \Psi_{K}\right)=\operatorname{proj}(\mathbf{x}, K)
$$




\section{REFERENCES}

1. J. C. Simo and R. L. Taylor, Consistent tangent operators of rate-independent elastoplasticity, Comp. Meth. Appl. Mech. Eng. 48: 101-118 (1985).

2. J. J. Moreau, Proximité et dualité dans un espace hilbertien, Bull. Soc. Math. France 93: 273-299 (1965).

3. J. J. Moreau, "On Unilateral Constraints, Friction and Plasticity," Lecture notes, CIME, Bressanone, 1973, Cremensese, Roma, 1971.

4. Nguyen Quoc Son, On the elastic plastic initial boundary value problem and its numerical integration, Int. J. Num. Meth. Eng. 11: 817-832 (1977).

5. P. Wriggers and J. C. Simo, A note on tangent stiffness for fully nonlinear contact problems, Comm. Appl. Num. Meth. 1: 199-203 (1985).

6. J. C. Simo, P. Wriggers, and R. L. Taylor, A perturbed Lagrangian formulation for the finite element solution of contact problems, Comp. Meth. Appl. Mech. Eng. 50: 163180 (1985).

7. A. Cumier and P. Alan, A generalized Newton method of contact problems with friction, J. Mec. Theory Appl. (1988).

8. P. Alart, "Multiplicateurs Augmentés et Méthode de Newton Généralisée Pour Contact avec Frottement," Rapport, Ecole Polytechnique Fédérale de Lausanne, Lausanne, Switzerland, 1988.

9. M. Jean, Dynamics with partially elastic shocks and dry friction: Double scale method and numerical approach, in Proceedings, 4th Meeting on Unilateral Problems in Structural Analysis, Capri, Italy, 1989.

10. Z. Mroz, Mathematical Models of Inelastic Material Behavior, University of Waterloo, Waterloo, Canada, 1973.

11. P. Germain, Mécanique des Milieux Continus, tome 1, Masson et Cie, Paris, 1973.

12. P. D. Panagiatopoulos, Inequality Problems in Mechanics and Applications, Convex and Nonconvex Energy Functions, Birkhauser, Boston, Basel, Stuttgan, 1985.

13. Nguyen Dang Hung, "Mécanique du Comportement Inélastique des Matériaux," Course Notes, Université de Liège, Liège, Belgium, 1980.

14. G. Duvaut and J. L. Lions, Les Inéquations en Mécanique et en Physique, Dunod, Paris, 1972.

15. Nguyen Dang Hung, "Sur la Plasticité et le Calcul des États Limites par Éléments Finis," Thèse de doctorat Spécial, Université de Liège, Liège, Belgium, 1984.

16. B. Halphen and Nguyen Quoc Son, Sur les matériaux standard généralisés, J. Mec. 14: 39-63 (1975).

17. J. Mandet, Cours de mécanique des milieux continus, tome 2, Mécanique des Solides, Gauthier-Villars, Paris, 1966.

18. P. Suquet, Local and global aspects in the mathematical theory of plasticity, in Plasticity Today, Modelling, Methods and Application, (A. Sawczuk and G. Bianchi, Eds.), Elsevier, London, 1983.

19. J. Lemaitre and J. L. Chaboche, Mécanique des Matériaux Solides, Bordas, Paris, 1985.

20. W. F. Chen, Limit Analysis and Soil Plasticity, Elsevier, New York, 1975.

21. Z. Mroz, Non-associated flow laws in plasticity. Mech. J. 2: 21-42 (1963).

22. P. D. Panagiatopoulos, A nonlinear programming approach for the unilateral contact and friction boundary value problem in the theory of elasticity, Ing. Arch. 44: 421-432 (1975).

23. M. Jean, "Contact Unilateral avec Frottement de Coulomb dans les Milieux Continus," Rapport GRECO No. 185/186, Université de Montpellier, Montpellier, France, 1985. 
24. M. Jean and G. Touzot, Implementation of unilateral contact and dry friction in computer codes dealing with large deformation problems, J. Theory Appl. Mech. 7: 145-160 (1988).

25. M. Jean, Unilateral contact with dry friction: Time and space discrete variables formulation, Actes du 6e Symposium Franco-Polonais sur la Mécanique Non-Lineaire, Villars de Lans, France, 1987.

26. M. Fortin and R. Glowinski, Méthodes de Lagrangien augmenté, application à la résolution numérique des problèmes aux limites, Coll. Meth. Math. Info. 9, Dunod, Paris, 1982.

27. S. C. Shyu, T. Y. Chang, and A. F. Saleeb, Friction contact analysis using a mixed finite element method, Comput. Struct. 32: 223-242 (1989).

28. M. Fremond, "Elude des Structures Visco-Élastiques Stratifiées Soumises à des Charges Harmoniques et des Solides Élastiques Reposant sur des Structures," Thèse, Université de Paris, Paris, 1971.

29. M. Fremond, Formulations duales des énergies potentielles et complémentaire, applications à la méthode des éléments finis, C.R. Acad. Sc. 1273 (serie A): 775-777 (1971).

30. M. Fremond, "Solid Resting on a Stratified Medium," presented at Int. Conf. on Varia. Meth. in Eng. Souphampton, UK, 1972.

31. P. Franchomme, A. Ricard, J. Oudin, and Y. Ravalard, Simulation du contact 2D dans SYSTUS par la méthode du Lagrangien augmenté, Proceed. Conf. STRUCOME (1989).

32. A. E. Giannakopoulos, The retum mapping method for the integration of friction constitutive relations, Comput. Siruct. 32: 157-167 (1989).

33. A. Klarbring and G. Bjorkman, A mathematical programming approach to contact problems with friction and varying contact surface, Comput. Struct. 30: 1185-1198 (1988).

34. W. X. Zhong and S. M. Sun, A parametric quadratic programming approach to elastic contact problems with friction, Comput. Struct. 32: 37-43 (1989).

35. G. De Saxce, "Sur Quelques Problèmes de Mécanique des Solides Considérés Comme Matériaux à Potentiels Convexes," Thèse de doctorat, Université de Liège, Liège, Belgium, 1986.

36. G. De Saxce and Nguyen Dang Hung, Dual analysis of frictionless problems by displacement and equilibrium finite elements, Eng. Struct. 6: 26-32 (1984).

37. Nguyen Dang Hung and G. De Saxce, Frictionless contact of elastic bodies by finite element method and mathematical programming technique, Comput. Struct. 11: 55-67 (1980).

38. Nguyen Dang Hung and G. De Saxce, Finite element analysis of contact problems based on the unilateral constraints formulation, in Structural Control (H. Leipholz, Ed.), NorthHolland, 1980, pp. 341-373.

39. I. Ekeland and R. Teman, Analyse Convexe es Problemes Variationnels, Dunod, Gauthiers-Villars, Paris, 1974.

40. P. Chabrand, Y. Pinto, and M. Raous, Numerical modeling of the friction in small and finite strains for metal forming processes: Analysis of the blankholder function, Euromech 273. Unilateral Contact and Dry Friction, Montpellier, France. May 1990.

41. L. Jeannin, "Etude mathématique et numérique d'un problème de contact unilatéral avec frottement en élasticité et élastoplasticité," Thèse de doctorat, Ecole centrale de Lyon, Paris (1985). 